\title{
SINDROME INTRA-SELAR
}

\author{
ANALISE DE 506 CIRURGIAS TRANSESFENOIDAIS
}

\author{
PEDRO SAMPAIO *
}

A sela túrcica, apesar de seu tamanho diminuto, constitui área de patologia peculiarmente importante. As lesões aí havidas, ao mesmo tempo que propiciam sérias desendocrinias, podem comprometer o sistema visual de modo irreversivel e fazer pressão no diafragma da sela levando a cefaléia persistente. Com base em nossa experiência, individualizamos uma "síndrome intra-selar", cuja existência não foi ainda devidamente valorizada. Esta sindrome se manifesta sobretudo em presença de tumores hipofisários, cistos intra-selares e sela vazia.

Os tumores hipofisários, quando do tipo embrionário, os craniofaringeomas, raramente se situam dentro da sela 6. A maioria tem localização supra-selar. Os adenomas, porém, nascem intra-selares e não raro ai se confinam, constituindo os microadenomas, pioneiramente estudados por Hardy 5 . As neoplasias da hipófise ganharam hoje classificação especial, graças à grande sensibilidade dos exames endócrinos 7. Assim é que, com base na produção ou não de hormônios específicos nomeiam-se: funcionantes e não funcionantes. Os tumores funcionantes compreendem: adenomas produtores de somatotrofina, manifestando-se por acromegalia; produtores de prolactina ou prolactinomas, exibindo a sindrome de galactorréia-amenorréia; produtores de ACTH com aumento de cortisol, compondo a sindrome de Cushing; os produtores de ACTH em doentes supra-adrenalectomizados, externando-se pela sindrome de Nelson e raros casos com produção de tireotrofina, expressando-se por hipertiroidismo secundário 1 . Os tumores não funcionantes manifestam-se apenas por compressão de estruturas vizinhas, vale dizer, comprometimento do quiasma óptico. O mesmo acontece com os cistos intra-selares 2 . A sela vazia constitui entidade a parte. A forma secundária aparece após cirurgia ou irradiação 10 e a primária tem sua origem em defeito congênito do diafragma da sela 3 . Encontra-se em cerca de 5\% das pessoas normais sem causar qualquer dano. Nos raros casos sintomáticos 9 , surge comprometimento visual ou hipopituitarismo discreto 4 .

\section{MATERIAL E METODO. RESULTADOS}

Nosso material proveniente da Universidade do Estado do Rio de Janeiro e da Clínica Santa Marta consta de 506 casos de cirurgias transesfenoidais, assim distribuidos:

Trabalho realizado no Serviço de Neurocirurgia da Universidade Estadual do Rio de Janeiro (UERJ): * Professor Titular de Neurocirurgia e Membro Titular da Academia Nacional de Medicina. 
396 tumores hipofisários, 4 cistos intra-selares, 4 selas vazias, 92 hipofisectomias totais e 8. reoperações (Tabela 1). Excluiremos deste estudo as hipofisectomias totais porque foram realizadas, não por patologia intra-selar, mas para alívio das dores de metástases ósseas de câncer da mama e da próstata. Serão também excluídas as fístulas liquóricas e as reoperações, por fugirem ao escopo deste trabalho. Os tumores estão assim distribuídos: não funcionantes, 120; secretantes de prolactina, 155; secretantes de somatotrofina, 80; secretantes de ACTH, 6; secretantes de ACTH com aumento de cortisol, 30; craniofaringeomas, 5 (Tabela 2).

Os 4 cistos intra-selares compunham-se de uma cápsula fibrosa tendo como conteúdo líquido xantocrômico. Três cram não comunicantes e um comunicante, conformando-se com os achados de Baskim (2). As 4 selas vazias eram do tipo primário. O quadro clínico ora se apresentou mono-sintomático ora mais de um sintoma acometeu o mesmo paciente. Encontramos 42 casos de hipopituitarismo, 62 de galactorréia-amenorréia, 93 de amenorréia, 80 de acromegalia, 30 de sindrome de Cushing, 6 de síndrome de Nelson e 102 pacientes com problemas visuais (Tabela 3). Todos os pacientes foram submetidos a exame endocrinológico.

Os exames complementares realizados foram: RX simples da sela túrcica, politomografia, tomografia computadorizada, angiografia cerebral e pneumocisternografia. $O$ critério de escolha dos exames baseou-se no quadro clínico apresentado. Todos os pacientes foram operados pelo autor por via transesfenoidal, havendo número muitó reduzido de complicaçc̃es (Tabela 4).

\begin{tabular}{lr}
\hline Cirurgias Transesfenoidais & Número de casos \\
\hline Tumores hipofisários & 396 \\
Cistos intra-selares & 4 \\
Sela vazia & 4 \\
Hipofisectomias totais & 92 \\
Fistulas liquóricas & 2 \\
Reoperações & 8 \\
Total & 506 \\
\hline
\end{tabular}

Tabela 1 - Cirurgias transesfenoidais em 506 casos.

Tumores hipofisários

\begin{tabular}{lr}
\hline Tumores hipofisários & Número de casos \\
\hline Não secretantes & 120 \\
Secretantes de prolactina & 155 \\
Secretantes de somatotrofina & 80 \\
Secretantes de ACTH & 6 \\
Secretantes de ACTH com aumento de cortisol & 30 \\
Craniofaringeomas & 5 \\
Total & 396 \\
\hline
\end{tabular}

Tabela 2 - Tipos de tumor hipofisdrio em 396 casos de sindrome intra-selaar. 


\begin{tabular}{lr}
\hline Quadro clínico & Número de casos \\
\hline Hipopituitarismo & 42 \\
Galactorréia-amenorréia & 62 \\
Amenorréia & 93 \\
Acromegalia & 80 \\
Sindrome de Cushing & 30 \\
Síndrome de Nelson & 6 \\
Sindrome visual & 102 \\
Cefaléla & 109 \\
Total & 404 \\
\hline
\end{tabular}

Tabela 3 - Quadro clinico em 404 pacientes com sindrome intra-selar.

\begin{tabular}{lc}
\hline Complicaços & Número de casos \\
\hline \hline Diabete insipido transitório & 6 \\
Rinoliquorréia & 6 \\
Meningite & 2 \\
Lesão da carótida & 1 \\
Lesão do seío cavernoso & 1 \\
Lesão do seio intercavernoso & 4 \\
Obito & 1 \\
\hline
\end{tabular}

Tabela 4 - Complicaçôes em 506 cirurgias transesfenoidais.

\section{COMENTARIOS}

Apesar da importância da "sindrome intra-selar" não tem a literatura corrente dado ênfase específica ao assunto 8 . Há descrições exaustivas de afecções intra-selares mas tem faltado um denominador comum para topografar a lesão. Concluimos de nosso material que existe uma triade que compõe a síndrome intra-selar: cefaléia, desendocrinias e desordens visuais. Até bem pouco tempo só se comprendia patologia intra-selar com aumento da sela, visto à radiologia simples. Hoje podemos fazer o diagnóstico de um adenoma hipofisário pelo simples perfil endocrinológico, mesmo com a sela de dimensões normais 11 . Por sua vez, o aumento da sela, nem sempre corresponde a patologia significativa. Pode tratar-se de sela vazia assintomática, achada por acaso ao se explorar um traumatismo de crânio ou buscar-se a causa de uma cefaléia pertinaz. Quanto às desordens do aparelho visual, nem sempre se delineia hemianopsia bitemporal com sua clássica simetria. Vezes há onde o campo visual tem características bizarras e necessita de exame apurado para se inferir da etiologia da lesão. Do nosso material concluimos que os tumores da hipófise contribuem com o maior contingente de patologia intra-selar, sendo as formas secretantes as mais frequentes. Entre estas, avultam os prolactinomas. A cefaléia foi o sintoma mais comum. Sabemos hoje que seu aparecimento se deve à compressão do diafragma da sela, de baixo para cima. Quando o diafragma se rompe 
e o tumor ultrapassa os limites da sela, geralmente a cefaléia se amaina. Em seguida, apareceram, como proeminentes, as desordens visuais. Se constituem o sintoma inicial, o paciente procura o oftalmologista e este deve estar alertado para a patologia intra-selar. Não é raro se encontrarem quadros graves e às vezes irreversíveis de deficiência visual, por falta de diagnóstico precoce. Queremos enfim nos reportar às desendocrinias que são os sintomas mais comuns em conjunto, embora não o sejam separadamente. Aos endocrinologistas compete então o diagnóstico. Mas, muitas vezes, no início da doença, o paciente procura o neurologista por cefaléia persistente, e a este compete, por indagação minuciosa, inteirar-se dos sintomas endócrinos.

Por constituir-se hoje a cirurgia transesfenoidal um tratamento simples e com mortalidade muito baixa, é de todo pertinente que se diagnostique precocemente a sindrome intra-selar. Mais ainda, já existem tratamentos clínicos promissores para controle de alguns adenomas hipofisários, ensejando portanto maior conforto terapêutico para tais pacientes.

\section{RESUMO}

Entre 506 cirurgias transesfenoidais realizadas pelo autor, foram selecionados para análise 404 casos assim distribuidos: 396 tumores hipofisários, 4 cistos intra-selares e 4 selas vazias. Com base nos sintomas apresentados pelos pacientes delineou-se a "síndrome intra-selar" caracterizada por cefaléia, desordens visuais e desendocrinias. Esta tríade pode às vezes aparecer incompleta. Chama-se atenção para casos de tumor intra-selar com RX da sela túrcica normal, onde o diagnóstico se faz apenas pelo perfil endocrinológico. Mostrá o autor que o reconhecimento da síndrome intra-selar pode ser feito precocemente se está atento para ela. Enfim o tratamento pela cirurgia transesfenoidal é simples e de excelentes resultados. A mortalidade na casuística do autor foi de $0,2 \%$.

\section{SUMMARY}

Intrasselar syndrome: analysis of 506 transsphenoidal surgeries.

The author has performed 506 transsphenoidal surgeries but has analyzed in this study only 404 cases. There were 396 hypophiseal tumors, 4 intrasellar cysts and 4 empty sellas. Based in the symptoms presented by the patients he describes an "intrasellar syndrome", caracterized by headache, visual disorders and hormonal abnormalities. He calls attention for intrasellar tumors with normal sella, as seen by $x$-ray. The diagnosis of these tumors were made only by endocrinological studies. At last he shows the advantages of transsphenoidal surgery in this pathology and the exellent results obtained. The mortality of his cases was only $0.2 \%$. 


\section{REFERENCIAS}

1. ASA, A.L. \& KALMAN, K. - Histological Classification of Pituitary Disease. Clinics in Endocrinology and Metabolism. Saunders, London, 1983, pg. 567.

2. BASKIN, D.S. \& WILSON, C.B. - Transsphenoidal treatment of non neoplastic intrasselar cysts. A report of 38 cases. J. Neurosurg. 60:8, 1984.

3. BUSCH, W. - Die Morphologie der Sella turcica and ihre Beziehungen zur Hypophyse. Virchows Arch. path. Anat. 320:437, 1951.

4. Empty sella and benign intrasselar cysts. Divitiis, E; Spaziante, R. \& Stella, I. - Advances and Technical Standards in Neurosurgery. Springer Verlag, Wien, 1981, pg. 1.

5. HARDY, J. - Transsphenoidal microsurgery of the normal and pathologic pituitary. Clin. Neurosurg. 16:185, 1969.

6. KOOS, W. Th. \& MILLER, M.H. - Intracranial Tumors of Infants and Children. G. Thieme, Sttutgart, 1971, pg. 199.

7. LANDOLT, Am. - Progress in Pituitary Adenoma Biology. Advances and Technical Standards in Neurosurgery. Springer Verlag, New York, 1978, pg. 1.

8. NEEDHAM, C.W. - Neurosurgical Syndromes of the Brain. Charles C. Thomas, Springfield, 1973.

9. OLSON, D.R.: GUIOT, D.D. \& DEROME, P. - The symptomatic empty sella. Prevention and correction via the transsphenoidal approach. J. Neurosurg. 37:533, 1972.

10. SACHDEV, Y.; EVERED, D.C.; APPLEBY, A. \& HALL, R. - Empty sella syndrome. Postgrad. med. J. 62:703, 1976.

11. THOMAS, J.P. \& HALL, R. - Medical Management of Pituitary Disease. Clinics in Endocrinology and Metabolism. Saunders, London, 1983, pg. 771.

Rua siāo João Batista, 80 - 22270, Rio de Janeiro, RJ - Brasil. 